\title{
Dynamics of seed magnetic island formation due to geometrically coupled perturbations
}

\author{
C. C. Hegna ${ }^{\text {a) }}$ and J. D. Callen \\ Departments of Engineering Physics and Physics, University of Wisconsin, Madison, Wisconsin 53706-1687
}

R. J. LaHaye

General Atomics, San Diego, California 92186

(Received 21 July 1998; accepted 29 September 1998)

\begin{abstract}
Seed magnetic island formation due to a dynamically growing external source in toroidal confinement devices is modeled as an initial value, forced reconnection problem. For an external source whose amplitude grows on a time scale quickly compared to the Sweet-Parker time of resistive magnetohydrodynamics, the induced reconnection is characterized by a current sheet and a reconnected flux amplitude that lags in time the source amplitude. This suggests that neoclassical tearing modes, whose excitation requires a seed magnetic island, are more difficult to cause in high Lundquist number plasmas. (C) 1999 American Institute of Physics. [S1070-664X(99)01901-1]
\end{abstract}

\section{INTRODUCTION}

A novel theoretical feature of neoclassical tearing modes in toroidal configurations is that a nonlinear excitation level is required for the destabilizing neoclassical effects to be activated. ${ }^{1-5}$ The source for the instability mechanism is the deformation of the perturbed bootstrap current profile in the vicinity of a sufficiently large "seed" magnetic island. The perturbed profile self-consistently enhances the seed island, producing magnetic perturbation, and ultimately produces a large macroscopic, sized magnetic island in high-temperature plasma, which can significantly limit plasma performance. ${ }^{6,7}$

A possible source for the seed island formation is the presence of a magnetic signal produced from another magnetohydrodynamic (MHD) event, such as a sawtooth crash or an edge localized mode (ELM) ${ }^{8,9}$ For example, associated with a sawtooth crash is a magnetic signal with poloidal and toroidal mode numbers $m=n=2$. In combination with toroidal geometry, this produces a $m=3, n=2$ magnetic signal that can, in principle, cause magnetic reconnection at the $q$ $=\frac{3}{2}$ surface. Experimental examination of the amplitude of the forced reconnection indicates a strong variation with the Lundquist number $S$. Specifically, the empirical observations of Ref. 8 report that the size of the seed island caused by a sawtooth crash scales as $\rho^{*} S^{-1}$, where $\rho^{*}$ is the normalized ion gyroradius. The role of this paper is to calculate the time dynamics of the forced reconnection at the driven resonance from a given source magnetic signal and to try to provide a possible explanation for the $S$ scaling of the seed island width.

The seed island formation as described above is an example of a forced reconnection. Forced magnetic reconnection is a subject of concern in magnetotail, coronal, and laboratory plasmas. In tokamaks, resonant magnetic field errors can produce localized current sheets and magnetic islands that can subsequently lead to dramatic changes in the bulk

${ }^{a)}$ Electronic mail: hegna@cptc.wisc.edu rotation profile and disruptions. ${ }^{10-13}$ Theoretical models have been developed to describe this phenomenon by accounting for the interaction of a resonant field error with a rotating resonant magnetic surface. ${ }^{14-16}$ These works differ in their treatment of the field error evolution. In Ref. 14, the field error is taken to be static and a steady-state reconnection response to that error is calculated. No temporal response is quantified. The approach of Ref. 14 differs from the work of Bhattacharjee and co-workers" 15,16 who treat the "Taylor problem" ${ }^{17,18}$ whereby a field error is impulsively turned on and the temporal evolution of the reconnected magnetic flux is calculated. A notable feature of this evolution is the presence of a "nonconstant- $\psi$ " phase where current sheets are formed. In this way, the temporal evolution of a forced reconnection more closely resembles the evolution properties of $m=1$ modes in tokamaks where the constant- $\psi$ assumption is invalid. ${ }^{19,20}$

Since the seed-producing magnetic perturbation is imagined to be produced from a temporally growing source, the theoretical treatment given here closely resembles the analytic model used to describe the Taylor problem. ${ }^{15-18} \mathrm{An}$ initial value problem is considered where we impose a particular time scale for the seed producing magnetic perturbation. If the imposed time scale is short enough, the reconnection process is described by a nonconstant- $\psi$ phase with a current sheet present at the rational surface. In this phase, the response at the rational surface causes the reconnected flux to lag in time the reconnected flux expected from simple geometric considerations with no layer physics response.

In the following section, we briefly introduce a simplified model to describe the interaction between an unstable temporally growing magnetic perturbation and a tearing stable magnetic perturbation that are coupled via geometric considerations. In Sec. III, we treat the linear evolution phase of the reconnection as an initial value problem. In Sec. IV, we consider the nonlinear evolution properties for a sufficiently large source magnetic perturbation amplitude. In Sec. $\mathrm{V}$, we include the effects of differential rotation in the non- 
linear regime. Finally, we discuss some implications in Sec. VI.

\section{BASIC MODEL}

We treat the problem of reconnection with asymptotic boundary layer analysis. The reconnection and concomitant magnetic island formation are assumed to occur in a localized region of space encompassing the rational magnetic surface. We calculate the details of the plasma response in this layer in the following sections. In the outer exterior region solution, the magnetic signals are governed by the ideal MHD equations at marginal stability conditions.

The MHD equilibrium is characterized by magnetic surfaces labeled by the "radial" variable $\rho$. The poloidal and toroidal angles are denoted $\theta$ and $\zeta$, respectively. For simplicity, a large aspect ratio tokamak ordering is used. The magnetic perturbations are written in the form $\delta \mathbf{B}=\nabla \zeta$ $\times \nabla \psi$, where $\psi$ is the magnetic flux function, which can be decomposed into Fourier harmonics of the poloidal and toroidal angles.

In this work, we are interested in the reconnection processes occurring at a rational surface caused to grow from an temporally growing external source. The external source models the existence of some other MHD event characterized by a growing magnetic perturbation. While general temporal growth is allowed for in some places, for specificity in the linear section, the external source magnetic signal is modeled to grow from time $t=0$ with the form

$$
\psi_{S}(t)=\Psi_{S}\left(\frac{t}{\tau_{G}}\right)^{\alpha},
$$

where $\Psi_{S}$ is the amplitude of the magnetic flux function associated with the magnetic signal, $\tau_{G}$ is the characteristic growth time of the signal, and $\alpha$ is some positive constant. Further, we assume that $\psi_{S}$ is described by a dominant helicity of the form $\psi_{S}=\psi_{S}(\rho) e^{i M \theta-i n \zeta}$ where $M$ and $n$ are the poloidal and toroidal mode numbers. In a toroidal equilibrium, $\psi_{S}$ is coupled via the geometry of the configuration to magnetic harmonics with common toroidal mode number of the form $\psi_{m}=\psi_{m}(\rho) e^{i m \theta-i n \zeta}$. We will be interested in a magnetic harmonic, which has a rational surface in the plasma at $\rho=\rho_{0}$ where the equilibrium safety factor satisfies $q\left(\rho_{0}\right)=m / n$.

The response of the magnetic fluctuation harmonic $\psi_{m}(\rho)$ to $\psi_{S}$ away from the rational surface is governed by the ideal MHD equations at marginal stability. That part of the exterior equations resonant with the mode numbers $m$ and $n$ yields an ordinary differential equation for $\psi_{m}(\rho)$ of the form

$$
\Lambda_{m} \psi_{m}(\rho)=-\Lambda_{S}^{m} \psi_{S}(\rho),
$$

where $\Lambda_{m}$ is the cylindrical exterior kink operator, which has second-order derivatives in $\rho$ and is singular at the rational surface $\rho=\rho_{0}$, and $\Lambda_{S}^{m}$ is an operator describing the geometric coupling between $\psi_{S}$ and $\psi_{m}$. The operators $\Lambda_{m}$ and $\Lambda_{S}^{m}$ are plasma profile and geometry dependent. Connor and coworkers have described explicit forms for these operators for the case of toroidicity coupling magnetic harmonics with mode numbers $m$ and $m \pm 1$ in a large aspect ratio ordering. ${ }^{21,22}$ The formalism introduced in Eq. (2) is identical to their treatment.

The general solution to (2) for $\psi_{m}(\rho)$ is given by

$$
\begin{aligned}
\psi_{m}(\rho) & =\Psi f_{m}^{-}(\rho)+g_{m}^{-}(\rho) \Psi_{S}\left(\frac{t}{\tau_{G}}\right)^{\alpha} \quad \text { for } \rho<\rho_{0} \\
& =\Psi f_{m}^{+}(\rho)+g_{m}^{+}(\rho) \Psi_{S}\left(\frac{t}{\tau_{G}}\right)^{\alpha} \quad \text { for } \rho>\rho_{0},
\end{aligned}
$$

where the dimensionless functions $f_{m}^{-}$and $f_{m}^{+}$satisfy the homogeneous equation $\Lambda_{m} f_{m}=0$ with boundary conditions $f_{m}^{-}\left(\rho_{0}\right)=f_{m}^{+}\left(\rho_{0}\right)=1, f_{m}^{-}(0)=0$ and $f_{m}^{+}(a)=0$ where $\rho=a$ is the location of a perfectly conducting wall. The functions $g_{m}^{-}$ and $g_{m}^{+}$satisfy Eq. (2) with boundary conditions $g_{m}^{-}(0)$ $=g_{m}^{-}\left(\rho_{0}\right)=g_{m}^{+}\left(\rho_{0}\right)=g_{m}^{+}(a)=0$. The value $\Psi$ represents the amount of reconnected magnetic flux at the rational surface $q=m / n$. The terms proportional to $\Psi_{S}$ represent the response of $\psi_{m}$ to the imposed source magnetic perturbation.

Due to the nature of the ideal MHD equations as the rational surface is approached, the exterior region solutions exhibit singular behavior at the rational surface. Namely, the general solution has a jump discontinuity in the perturbed magnetic flux across the rational surface. For simplicity, a small $\beta$ ordering $\left(\beta=2 \mu_{0} p / B^{2} \ll 1\right)$ is used in the exterior region, however, the results can be easily generalized to the finite- $\beta$ case. This discontinuity is quantified by the parameter $\Delta^{\prime}$ of tearing mode theory, ${ }^{23}$ where

$$
\Delta^{\prime}=\left.\lim _{\epsilon \Rightarrow 0} \frac{1}{\psi_{m}\left(\rho_{0}\right)} \frac{d \psi_{m}}{d r}\right|_{\rho_{0}-\epsilon} ^{\rho_{0}+\epsilon},
$$

Using Eq. (3), $\Delta^{\prime}$ can be written as the sum of two terms

$$
\Delta^{\prime}=\Delta_{\text {mode }}^{\prime}+\Delta_{c}^{\prime} \frac{\psi_{S}(t)}{\Psi},
$$

where the first term is given by

$$
\Delta_{\text {mode }}^{\prime}=\frac{d f_{m}^{+}}{d r}\left(\rho_{0}\right)-\frac{d f_{m}^{-}}{d r}\left(\rho_{0}\right),
$$

which represents the tearing mode stability parameter of the mode at the rational surface $q=m / n$ in the absence of the external source perturbation. In this work, we assume that the $\psi_{m}$ is tearing stable $\left(\Delta_{\text {mode }}^{\prime}<0\right)$ and that the source for magnetic reconnection is due to the forcing term given by the last term in Eq. (5) with

$$
\Delta_{c}^{\prime}=\frac{d g_{m}^{+}}{d r}\left(\rho_{0}\right)-\frac{d g_{m}^{-}}{d r}\left(\rho_{0}\right) .
$$

The physical interpretation of a $\Delta^{\prime}$ is that it is proportional to the value of the current sheet $\delta J_{\zeta}=\delta \mathbf{J} \cdot \mathbf{e}_{\zeta}$ $=\nabla^{2} \psi_{m} / \mu_{0}$ at the rational surface

$$
\begin{aligned}
\delta J_{\zeta} \mu_{0} & \cong \delta\left(\rho-\rho_{0}\right) \Delta^{\prime} \Psi \\
& =\delta\left(\rho-\rho_{0}\right)\left[\Delta_{\text {mode }}^{\prime} \Psi+\Delta_{c}^{\prime} \psi_{S}(t)\right] \\
& \cong-\delta\left(\rho-\rho_{0}\right) \frac{2 m}{\rho_{0}}[\Psi-E(t)],
\end{aligned}
$$


where the last form is obtained using the approximation $\Delta_{\text {mode }}^{\prime}=-2 m / \rho_{0}$ and defining $E(t)=\psi_{S}(t)\left(\rho_{0} \Delta_{c}^{\prime} / 2 m\right)$. Even in the absence of any magnetic reconnection $(\Psi=0)$, the source term still causes the formation of a singular current sheet at the rational surface. The introduction of plasma resistivity in the layer region will then allow the current sheet to relax and lead to a forced magnetic reconnection.

In the inner region solution, the singular current is resolved by including nonideal MHD effects. Asymptotic matching between the inner region and exterior region yields predictions for the evolution properties of the mode evolution. This is explicitly calculated by the matching condition

$$
\Delta^{\prime} \Psi=-\frac{2 m}{\rho_{0}}[\Psi-E(t)]=\int_{-\infty}^{+\infty} d x \mu_{0} J_{\text {inner }}(x),
$$

where $\mu_{0} J_{\text {inner }}=\nabla^{2} \psi_{\text {inner }}$ is the resolved inner region solution resonant with the magnetic perturbation, describing the current profile near the rational surface on the inner strained variable $x=\rho-\rho_{0}$.

If there is no response at the rational surface, $J_{\text {inner }} \Rightarrow 0$, the reconnection is predicted to proceed by the relation $\Psi$ $=E(t)$, which states that the reconnected flux grows directly with the source perturbation with a proportionality constant $\rho_{0} \Delta_{c} / 2 m$, which is solely a function of the equilibrium and geometric properties. However, as is demonstrated in the following sections, the layer response is important in describing the reconnection process and, in general, the simple geometric argument, $\Psi=E(t)$ is not valid.

\section{LINEAR THEORY}

In this work, the low- $\beta$ reduced resistive MHD equations are used for simplicity. The equilibrium magnetic field is approximately described in the vicinity of the rational surface $\rho=\rho_{0}$ by the form

$$
\mathbf{B}_{0}=B_{\zeta}\left(\nabla \zeta+\nabla \zeta \times \nabla \frac{x^{2}}{L_{s}}\right),
$$

where $B_{\zeta}$ is a constant, $x=\rho-\rho_{0}$, and $L_{s}$ is the magnetic shear length, which in the high aspect ratio tokamak ordering is $L_{s}=q_{0}^{2} R / q_{0}^{\prime} \rho_{0}$, where the major radius $R$, safety factor $q_{0}$ and its derivative $q_{0}^{\prime}$ are all evaluated at the rational surface. The helically resonant angle is written $u=\theta-\zeta / q_{0}$. The perturbed magnetic and velocity fields are described using the potentials $\psi$ and $\varphi$, respectively, with

$$
\begin{aligned}
& \delta \mathbf{B}=\nabla \zeta \times \nabla \psi, \\
& \delta \mathbf{v}=\nabla \zeta \times \nabla \varphi,
\end{aligned}
$$

which guarantees $\nabla \cdot \delta \mathbf{B}=0$ and incompressibility, $\nabla \cdot \delta \mathbf{v}$ $=0$. The governing equations are Ohm's law and quasineutrality given by

$$
\begin{aligned}
& \frac{\partial \psi}{\partial t}=\mathbf{B} \cdot \nabla \varphi+\frac{\eta}{\mu_{0}} \nabla^{2} \psi, \\
& \rho_{M} \mu_{0}\left(\frac{\partial}{\partial t}+\mathbf{v} \cdot \nabla\right) \nabla^{2} \varphi=\mathbf{B} \cdot \nabla\left(\nabla^{2} \psi\right),
\end{aligned}
$$

where $\eta / \mu_{0}$ is the resistive diffusivity, $\rho_{M}$ is the mass density and $\mathbf{B}=\mathbf{B}_{0}+\delta \mathbf{B}$.

Linearizing Eqs. (13) and (14) using $\psi=\psi(x) e^{\mathrm{imu}}, \varphi$ $=\varphi(x) e^{\mathrm{imu}}$, and the small layer width assumption $\partial / \partial x$ $\gg m / \rho_{0}$, gives the equations

$$
\begin{aligned}
& \frac{\partial \psi}{\partial t^{\prime}}=i x^{\prime} \varphi^{\prime}+\hat{\eta} \frac{d^{2} \psi}{d x^{\prime 2}}, \\
& \frac{\partial}{\partial t^{\prime}} \frac{d^{2} \varphi^{\prime}}{d x^{\prime 2}}=i x^{\prime} \frac{d^{2} \psi}{d x^{\prime 2}},
\end{aligned}
$$

where the normalizations $t^{\prime}=t / \tau_{a}, \quad x^{\prime}=m x / \rho_{0}$ and $\varphi^{\prime}$ $=\varphi\left(\rho_{M} \mu_{0}\right)^{1 / 2}$ are used in which $\tau_{a}=L_{s} / v_{a}$ $=R L_{s}\left(\rho_{M} \mu_{0}\right)^{1 / 2} / B_{\zeta}$ and $\hat{\eta}=\left(\eta / \mu_{0}\right)\left(m^{2} L_{s} / v_{a} \rho_{0}^{2}\right)$.

We will be considering an initial value problem where at time $t=0$ the exterior source grows as defined in Eq. (1) and there is no reconnection at $x=0$ consistent with the assumed inherent tearing stable properties of the mode. It is convenient to Fourier and Laplace transform the layer equations using

$$
\begin{aligned}
& \hat{\psi}(p, k)=\int_{0}^{\infty} d t^{\prime} \int_{-\infty}^{+\infty} d x^{\prime} e^{-p t^{\prime}} e^{i k x^{\prime}} \psi\left(x^{\prime}, t^{\prime}\right), \\
& \psi(x, t)=\mathscr{D}^{-1} \int_{-\infty}^{+\infty} \frac{d k}{2 \pi} e^{-i k x} \hat{\psi}(p, k),
\end{aligned}
$$

where $\mathscr{B}^{-1}$ is the inverse Laplace transform and appropriate boundary conditions are imposed. Equations (15) and (16) can be combined to yield a linear eigenmode equation for the transformed current density, $\hat{J}=-k^{2} \hat{\psi}$

$$
k^{2} \frac{d}{d k} \frac{1}{k^{2}} \frac{d \hat{J}}{d k}=\left(p^{2}+p \hat{\eta} k^{2}\right) \hat{J},
$$

which has a solution in terms of Whittaker functions

$$
\hat{J}(k, p)=J_{0} k^{1 / 2} W_{-\lambda, 3 / 4}(z),
$$

where $\lambda=p^{3 / 2} / 4 \hat{\eta}^{1 / 2}, z=k^{2} \sqrt{p \hat{\eta}}$ and $J_{0}$ is a constant.

The evolution of the reconnected flux $\Psi(t)$ is governed by Ohm's law at $x=0$

$$
\frac{\partial \Psi}{\partial t}=\frac{\eta}{\mu_{0}} J(x=0)=\frac{\eta}{2 \pi \mu_{0}} \int_{-\infty}^{+\infty} d k \mathscr{B}^{-1} \hat{J} .
$$

Laplace transforming the matching condition, Eq. (9) gives an equation for $\hat{\Psi}$, the Laplace transform of $\Psi(t)$

$$
E_{0} \frac{\Gamma(1+\alpha)}{\tau_{G}^{\alpha} p^{\alpha+1}}-\hat{\Psi}=\frac{p \pi \tau_{r}}{m^{2}} \hat{\Psi} \frac{\hat{J}(k=0, p)}{\int_{-\infty}^{+\infty} d k \hat{J}},
$$

where $E(t)=E_{0}\left(t / \tau_{G}\right)^{\alpha}$ characterizes the temporal evolution of the source and $\tau_{r}=\rho_{0}^{2} \mu_{0} / \eta$ is the resistive diffusion time.

One can now evaluate the time dynamics of the reconnected flux by evaluating Eq. (21) using Eq. (19) and inverting the transforms. Different asymptotic regimes in time appear. At early times $t<\tau_{a}^{2 / 3} \tau_{r}^{1 / 3}=\tau_{a} S^{1 / 3}$, where $S=\tau_{r} / \tau_{a}$ is an effective Lundquist number here, Eq. (21) gives

$$
p^{2} \frac{\pi \tau_{a} \tau_{r}}{2 m^{2}} \hat{\Psi}=E_{0} \frac{\Gamma(1+\alpha)}{\tau_{G}^{\alpha} p^{\alpha+1}}-\hat{\Psi} .
$$


In this limit, resistivity plays no role in establishing the characteristic time and length scales of the layer, $\lambda \gg 1$. However, resistivity is clearly needed for magnetic reconnection to proceed. In this limit the last term in Eq. (22) can be neglected and the reconnection flux grows in time with

$$
\begin{aligned}
\Psi(t) & =E_{0} \frac{t^{2+\alpha}}{\tau_{G}^{\alpha} \tau_{a}^{2}} \frac{2 m^{2}}{\pi(\alpha+1)(\alpha+2)} \\
& =\frac{t^{2}}{\tau_{s p}^{2}} E(t) \frac{2 m^{2}}{\pi(\alpha+1)(\alpha+2)},
\end{aligned}
$$

where $\tau_{s p}=\sqrt{\tau_{a} \tau_{r}}$ is the Sweet-Parker time scale. We note that the Taylor problem result as originally formulated can be obtained from Eq. (23) by taking $\alpha=0$ with $E(t)=E_{0} \cdot{ }^{17,18}$ The important thing to be learned from Eq. (23) is that on this time scale $\left(t<\tau_{a} S^{1 / 3}\right)$, the reconnected flux amplitude lags $E(t), \Psi \ll E(t)$. From the layer equation, the characteristic linear layer width in this phase can be deduced from the scaling $\delta_{L}=\Delta B_{\perp} / J(0)$

$$
\delta_{L}=\rho_{0} \pi \frac{\alpha+1}{m} \frac{\tau_{a}}{t},
$$

where $\Delta B_{\perp}$ is the jump in the perpendicular magnetic field across the layer. Further the quantity

$$
\Delta^{\prime} \delta_{L} \cong \frac{\tau_{r} \tau_{a}^{2}}{t^{3}} \frac{\pi^{2}(\alpha+1)^{2}(\alpha+2)}{m^{2}} \gg 1,
$$

is large denoting that there is rapid variation in $\psi(x)$ in the layer. Equation (25) shows that the linear mode growth initially ensues in a nonconstant- $\psi$ phase, as has been shown previously, ${ }^{17,18}$ which differs from standard linear tearing mode theory. ${ }^{23}$ In this phase, a singular current of amplitude $J(0) \cong\left(t / \tau_{a}\right) E(t)\left[2 m^{2} / \pi \rho_{0}^{2}(\alpha+1)\right]$ grows in the layer.

On longer time scales, resistive effects enter into the layer physics. On time scales in the range, $\tau_{a} S^{1 / 3} \ll t$ $\ll \tau_{a} S^{3 / 5}$, the dominant terms in Eq. (21) are given by

$$
\left(p \tau_{\mathrm{FKR}}\right)^{5 / 4} \hat{\Psi}=E_{0} \frac{\Gamma(1+\alpha)}{\tau_{G}^{\alpha} p^{\alpha+1}},
$$

where

$$
\tau_{\mathrm{FKR}}=\frac{\tau_{r}^{3 / 5} \tau_{a}^{2 / 5}}{m^{6 / 5}}\left[\pi \frac{\Gamma(3 / 4)}{\Gamma(1 / 4)}\right]^{4 / 5},
$$

is the characteristic growth time of the linear tearing mode. ${ }^{23}$ Inverting Eq. (26) gives the linear growth phase in this asymptotic range

$$
\begin{aligned}
\Psi(t) & =\frac{t^{\alpha+5 / 4}}{\tau_{\mathrm{FKR}}^{5 / 4} \tau_{G}^{\alpha}} E_{0} \frac{\Gamma(\alpha+1)}{\Gamma(\alpha+5 / 4)} \\
& =\left(\frac{t}{\tau_{\mathrm{FKR}}}\right)^{5 / 4} E(t) \frac{\Gamma(\alpha+1)}{\Gamma(\alpha+5 / 4)} .
\end{aligned}
$$

For time scales short of $\tau_{\mathrm{FKR}}, \Psi(t)$ still lags $E(t)$. The linear layer width is given by

$$
\delta_{L} \cong \frac{\rho_{0}}{\sqrt{m}} \frac{\tau_{a}^{1 / 2}}{\tau_{r}^{1 / 4} t^{1 / 4}} k_{0}
$$

and

$$
\Delta^{\prime} \delta_{L} \cong \frac{\tau_{a} \tau_{r}^{1 / 2}}{t^{3 / 2}} \frac{k_{1}}{m},
$$

where $k_{0}$ and $k_{1}$ are positive functions of $\alpha$. Equation (30) indicates that the mode makes a transition from a nonconstant- $\psi$ phase $\left(\Delta^{\prime} \delta_{L} \gtrsim 1\right)$ to a constant- $\psi$ phase $\left(\Delta^{\prime} \delta_{L} \ll 1\right)$ on time scales long compared to $\tau_{a} S^{1 / 3}$.

If the characteristic time of interest is longer than $\tau_{\mathrm{FKR}}$, the first term in Eq. (9) is no longer negligible and the reconnection rate $\Psi(t) \cong E(t)$ is obtained. In this limit the source growth is slow enough that the perturbed current in the layer has had sufficient time to relax and the mode grows on the slow time scale of the geometrically coupled mode.

However, all the assertions made in this section concerning mode evolution time scales and responses are based upon the use of linear theory. As a matter of practical importance, this phase may have very little to do with the actual mode dynamics, since nonlinear effects are known to significantly modify tearing mode growth at very small fluctuation level (essentially, the nonlinearities come into play when the island width is comparable to the linear layer widths). We address this issue in the following section.

\section{NONLINEAR RESPONSE}

As shown by Wang and Bhattacharjee, for a sufficiently large forced reconnection amplitude, a nonlinear phase follows linear growth that is characterized by current sheets. ${ }^{16,18}$ In this regime, a variant of Sweet-Parker reconnection theory is used to describe the mode evolution. ${ }^{19,24,25}$

Nonlinear effects become important when the linear and nonlinear parts of the last term in Eq. (14) become comparable. Crudely, this is the case when the perturbation amplitude $\psi$ is of the same order as $\delta^{2} B_{\zeta} / L_{s}$ where $\delta$ is the typical scale length of the layer. Using $\psi \cong \Psi+E \delta\left(2 \mathrm{~m} / \rho_{0}\right)$ yields the onset condition in the nonconstant $\psi$ regime as $2 \mathrm{mE} / \Phi$ $\cong\left(\delta / \rho_{0}\right)$, where $\Phi=B_{\zeta} \rho_{0}^{2} / L_{s}$. Using the linear layer estimate for $\delta$ from Eq. (24), this gives the condition for onset of nonlinear effects as $2 m E / \Phi \cong \tau_{a} / t$ which is of the order $S^{-1 / 3}$ in the nonconstant- $\psi$ regime. On longer time scales, the condition for nonlinear onset becomes less stringent. For present day high-temperature tokamak experiments with $S$ $\cong 10^{8}-10^{9}$, the nonlinear regime is of interest.

In this nonlinear regime, assuming $\partial / \partial x \gg m / \rho_{0}$, reconnection occurs at the approximate rate

$$
\frac{\partial \psi_{x}}{\partial t}=\frac{\eta}{\mu_{0}} \frac{\partial^{2} \psi}{\partial x^{2}} \cong \frac{\eta}{\mu_{0}} \frac{R \Delta B_{\perp}}{\delta_{N L}},
$$

where $\psi=\psi_{x}$ is the separatrix where $\mathbf{v} \cdot \nabla \psi \cong 0, \Delta B_{\perp}$ is the jump in the perpendicular magnetic field across the rational surface, and $\delta_{N L}$ is the current layer width in this regime. Using the properties of mass conservation and momentum balance, one obtains a condition for the plasma flow into the reconnection region $v_{x}$

$$
v_{x}=\frac{\delta_{N L}}{\rho_{0}} v_{y},
$$


where $v_{y} \cong \Delta B_{\perp} / \sqrt{\rho_{M} \mu_{0}}$ is the Alfvén velocity associated with the reconnected flux. Outside the layer, the plasma is ideal, which gives

$$
\frac{\partial \psi_{x}}{\partial t} \cong v_{x} \Delta B_{\perp}
$$

Equations (31)-(33) can be combined to produce a relation for the nonlinear layer width, given by

$$
\delta_{N L}=\sqrt{\frac{\eta \rho_{0}}{\mu_{0} v_{y}}} .
$$

Using Eq. (34) in Eq. (31) and $\Delta B_{\perp}=2 m E(t) / R \rho_{0}$, one obtains

$$
\psi_{x}(t)=\int_{0}^{t} d t^{\prime} \frac{1}{\tau_{s p}} \frac{\left[2 m E\left(t^{\prime}\right)\right]^{3 / 2}}{\sqrt{\Phi}} .
$$

One can modify the MHD model by including a crossfield viscosity in the momentum balance equation of the form $v \rho_{M} \nabla^{2} \mathbf{v}$. This effect slows down the reconnection rate. ${ }^{19}$ In the presence of viscosity, the reconnection time scale is replaced by

$$
\tau_{s p v}=\tau_{s p}\left(1+\frac{v \mu_{0}}{\eta}\right)^{1 / 4}
$$

As in the linear case, on time scales smaller than the Sweet-Parker time, the reconnected flux lags behind $E(t)$. In this regime, there is a significant plasma response in the layer. On longer time scales, the highly singular current layer response decays and the nonlinear evolution is determined by a modified Rutherford regime. ${ }^{26}$ This regime describes an evolution equation for the nonlinear island width $w$ $=4 \sqrt{\Psi L_{s} / B_{T}}$

$$
\frac{d w}{d t}=1.22 \frac{\eta}{\mu_{0}} \Delta^{\prime}=1.22 \frac{\eta}{\mu_{0}} \frac{2 m}{\rho_{0}}\left[\frac{E(t)}{\Psi}-1\right],
$$

which again predicts $\Psi=E(t)$ in "saturation."

\section{EFFECT OF DIFFERENTIAL ROTATION}

If the magnetic surface where the forced reconnection occurs is rotating relative to the magnetic signal, the induced island width is reduced. ${ }^{14,16}$ Differential rotation can be modeled by modifying the source temporal evolution. In this section, a source term of the form

$$
E(t)=E_{0}\left(\frac{t}{\tau_{G}}\right)^{\alpha} e^{i \omega t},
$$

is used where $\omega$ is the relative rotation rate.

In the nonlinear regime, the induced current sheet at the rational surface rotates relative to the rational surface. A modified Sweet-Parker analysis gives the relation ${ }^{16}$

$$
\frac{\partial \psi_{x}}{\partial t}=\frac{e^{i \omega t}}{\tau_{s p}} \frac{[2 m E(t)]^{3 / 2}}{\sqrt{\Phi}} .
$$

For $\omega t \ll 1$, Eq. (39) gives

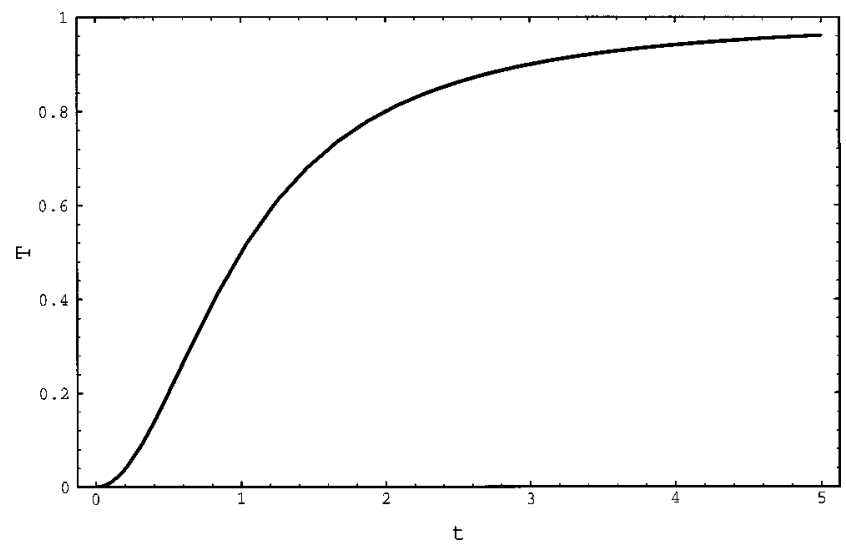

FIG. 1. The electromagnetic torque (normalized to its peak value $T$ $=T_{E M \zeta} / T_{\infty}$ ), as a function of time (normalized by $\omega \sqrt{C_{1}}$ ) for fixed differential rotation frequency $\omega$. At large time, $\omega t \sqrt{C_{1}} \gg 1$, the torque obtains its largest value $T_{E M \zeta}=T_{\infty}=\left(\omega \tau_{s p}\right)^{-1}\left(8 n \pi^{2} / R\right)(2 m E)^{5 / 2} \Phi^{-1 / 2}$.

$$
\psi_{x}(t) \cong \frac{t}{\tau_{s p}} \frac{[2 m E(t)]^{3 / 2}}{\sqrt{\Phi}} \frac{1}{1+3 \alpha / 2}\left\{1-i \omega t C_{0}+\cdots\right\}
$$

where Eq. (1) is used and $C_{0}=(1+3 \alpha / 2) /(2+3 \alpha / 2)$. In this $\omega t \ll 1$ regime, the reconnection ensues as in the previous section and rotation effects enter at higher order. However, in the asymptotic regime $\omega t>1$, Eq. (39) gives

$$
\psi_{x}(t) \cong \frac{e^{i \omega t}}{i \omega \tau_{s p}} \frac{[2 m E(t)]^{3 / 2}}{\sqrt{\Phi}}\left[1+O\left(\frac{1}{\omega t}\right)\right],
$$

which shows a reduction in the reconnected flux from that given by Eq. (35) by the small factor $1 / \omega t$. In this limit, the differential rotation has prevented significant reconnection.

The presence of an imaginary response in the mode evolution equation indicates a phase slippage between the current sheet and the reconnected flux. This produces a net electromagnetic force at the rational surface through the nonlinear $\mathbf{J} \times \mathbf{B}$ force. Following Fitzpatrick, ${ }^{14}$ we can define the electromagnetic torque in the toroidal direction

$$
\begin{aligned}
T_{E M \zeta} & =\int \rho d \rho \int d \theta \int d \zeta R^{2} \hat{\zeta} \cdot \mathbf{J} \times \mathbf{B} \\
& =8 \pi^{2} \frac{m n}{R}\left(i \Psi E^{*}-i E \Psi^{*}\right),
\end{aligned}
$$

where the radial integration encompasses a small region near the rational surface. Using $\Psi=\psi_{x}$, an approximate expression for Eq. (42) can be obtained that describes the two asymptotic limits of Eqs. (40) and (41), which is given by

$$
T_{E M \zeta} \cong 8 \pi^{2} \frac{n}{R} \frac{[2 m E(t)]^{5 / 2}}{\sqrt{\Phi}} \frac{1}{\tau_{s p}} \frac{C_{1} \omega t^{2}}{\left(1+C_{1} \omega^{2} t^{2}\right)},
$$

where $C_{1}=\left(C_{0}+1\right) /(1+3 \alpha / 2)$. As shown in Fig. 1, for a given rotation frequency, the electromagnetic torque grows in time and saturates in the $\omega t \gg 1$ asymptotic limit. The large time limit is equivalent to that given in Ref. 16. In Fig. 2 , a plot of electromagnetic torque as a function of $\omega$ is given for a fixed time. The electromagnetic torque goes to zero in 


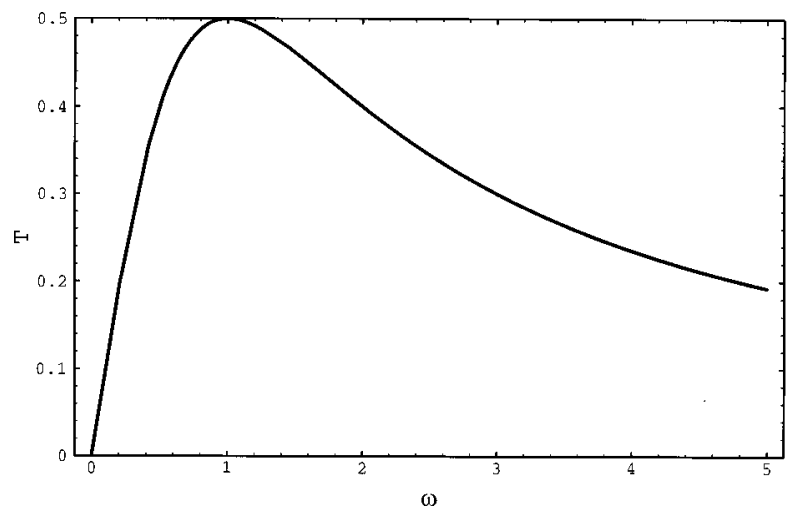

FIG. 2. The electromagnetic torque [normalized to the value $T_{1}=\left(t / \tau_{s p}\right)$ $\left.\times\left(8 n \pi^{2} \sqrt{C_{1}} / R\right)(2 m E)^{5 / 2} \Phi^{-1 / 2}\right]$ as a function of frequency (normalized to $t \sqrt{C_{1}}$ ) for a particular value of time. In this plot, the torque obtains its peak value at $\omega t \sqrt{C_{1}}=1$ and goes to zero in the asymptotic limits $\omega \Rightarrow 0$ and $\omega \Rightarrow \infty$.

the two extreme limits of $\omega \Rightarrow 0$ and $\omega \tau_{s p} \Rightarrow \infty$. The peak torque as a function of frequency is given by the condition $\omega t \sqrt{C_{1}}=1$.

As has been shown in Refs. 14 and 16, a model for mode locking can be constructed by balancing the plasma inertia and viscous torques against the electromagnetic torques of Eq. (43). In the $\omega t \gg 1$, this procedure yields the results of Ref. 16 where the nonconstant- $\psi$ Sweet-Parker theory was used. If the characteristic time scale of the source is slower than the Sweet-Parker rate, reconnection occurs on the slower Rutherford scale and the effect of differential rotation can be calculated using the constant- $\psi$ theory of Ref. 14 .

\section{DISCUSSION}

A commonly used assertion concerning the initiation of neoclassical MHD tearing instabilities is that some other MHD event produces a small seed island that allows the destabilizing neoclassical effects to produce macroscopicsized magnetic islands. The results of the calculation presented here is that simple geometric arguments for calculating the seed island amplitude are not generally correct if the time dynamics of the forced reconnection process are properly accounted for. If the magnetic signal responsible for the seed island formation grows and decays on a time scale short compared to the Sweet-Parker time, no appreciable forced reconnection occurs. Two commonly observed MHD events in tokamaks where these time scale arguments are applicable are sawteeth and ELMs. Present day observations of sawteeth in tokamaks indicate that the crash times are quicker than the Sweet-Parker time scale. The magnetic signal associated with ELMs tends to be on the ideal MHD time scale, which is also much quicker than the characteristic forced reconnection time. Conversely, resistive tearing modes grow on much slower time scales. ${ }^{26}$ Consequently, appreciable forced reconnection can be caused by slowing growing tearing instabilities. The theory for this case, where the constant- $\psi$ assumption is valid, has been worked in detail in Ref. 27.
Quantitative estimates for the amplitude of the reconnected flux in the experimentally relevant nonlinear regime are given in Eq. (35). For a given value of a source magnetic perturbation, the induced mode amplitude scales as the ratio of the characteristic growth time divided by the SweetParker time. If the characteristic time scale of the source is independent of resistivity (as in an ideal MHD mode), Eq. (35) predicts that the induced amplitude decreases with Lundquist number, $\psi_{x} \sim S^{-1 / 2}$. If the cross-field viscosity is included, [see Eq. (36)], and $\nu>\eta / \mu_{0}$, which is generally true in high-temperature tokamaks, the scaling is modified to be $\psi_{x} \sim R^{1 / 4} S^{-3 / 4}$, where $R / S=\nu \mu_{0} / \eta$. This scaling indicates that for a given source amplitude, it becomes increasingly difficult to initiate a seed magnetic island with increasing Lundquist number.

Differential rotation also has the effect of reducing the forced reconnection amplitude. From Eq. (41), valid when $\omega t>1$, the reconnected flux scales as $1 / \omega \tau_{s p v}$ for a given source amplitude. For rotation rates independent of resistivity, the same scaling laws of $\Psi$ with $S$ are obtained as described in the previous paragraph.

While the prediction that the seed island amplitude decreases with $S$ is in qualitative agreement with the experimental observations, ${ }^{8}$ a quantitative discrepancy still remains. However, open experimental and theoretical issues are present that require further study. Initial experimental studies indicated a seed island width that scales as $\rho^{*} S^{-1}$. This scaling is essentially empirical and more data is required to make definitive statements on the $S$ scaling. Since the island width needs to exceed the ion banana width for mode growth to ensue, ${ }^{1}$ there may be a bias toward having the $\rho^{*}$ scaling. There is no obvious reason that the ion gyroradius need enter into the seed island formation process. Further studies of the experimental data suggests a weaker dependence on $S$ than reported in Ref. 8. This result will be reported elsewhere. In addition to the experimental issues, there are also open theoretical questions. In particular, there may be an additional $S$ scaling in the source amplitude, $\Psi_{s}$, which depends upon the nonlinear physics associated with the appearance of the source magnetic perturbation. In order to completely predict the entire behavior of the coupled seed island problem, one would need to have a more accurate sawtooth or ELM model to predict the $\Psi_{s}$. The conclusions of the present work are based solely upon predicting the relationship between a seed island to a given magnetic source.

A key theoretical aspect of the calculation was the necessity of accounting for the current sheet in the reconnection layer. For sufficiently fast time scales, reconnection occurs in a nonconstant- $\psi$ phase. As noted previously, in this way the forced reconnection problem resembles the growth of $m=1$ modes in tokamaks. ${ }^{20}$ It is generally recognized that resistive MHD does not accurately describe the fast crash times of sawteeth and that collisionless effects need to considered. ${ }^{28,29}$ Since the physics of the forced reconnection and the $m=1$ mode is similar, collisionless effects may also be necessary to accurately predict the seed island formation in the problem presented here. We leave this open for future work. 


\section{ACKNOWLEDGMENTS}

The authors acknowledge useful conversations with Tom Gianakon on this subject. This work is supported by the U.S. Department of Energy under Grant No. DE-FG0292ER54139 and Contract No. DE-AC03-89ER51114.

${ }^{1}$ H. R. Wilson, J. W. Connor, R. J. Hastie, and C. C. Hegna, Phys. Plasmas 3, 248 (1996).

${ }^{2}$ R. Fitzpatrick, Phys. Plasmas 2, 825 (1995).

${ }^{3}$ N. N. Gorolenkov, R. V. Budny, Z. Chang, M. V. Gorelenkova, and L. E. Zakharov, Phys. Plasmas 3, 3379 (1996).

${ }^{4}$ T. A. Gianakon, J. D. Callen, and C. C. Hegna, Phys. Plasmas 3, 4637 (1996).

${ }^{5}$ C. C. Hegna, Phys. Plasmas 5, 1767 (1998).

${ }^{6}$ Z. Chang, J. D. Callen, C. C. Hegna, E. D. Fredrickson, R. V. Budny, K. M. McGuire, M. C. Zarnstorff, and the TFTR Group, Phys. Rev. Lett. 74, 4663 (1994).

${ }^{7}$ O. Sauter, R. J. La Haye, Z. Chang, D. A. Gates, Y. Kamada, H. Zohm, A. Bondeson, D. Boucher, J. D. Callen, M. S. Chu, T. A. Gianakon, R. W. Harvey, C. C. Hegna, L. L. Lao, D. A. Monticello, F. Perkins, A. Pletzer, A. H. Reiman, M. Rosenbluth, E. J. Strait, T. S. Taylor, A. D. Turnbull, F. Waelbroeck, J. C. Wesley, H. R. Wilson, and R. Yoshino, Phys. Plasmas 4, 1654 (1997), and references within.

${ }^{8}$ R. J. LaHaye and O. Sauter, Nucl. Fusion 38, 1 (1998).

${ }^{9}$ T. A. Gianakon, C. C. Hegna, and J. D. Callen, Bull. Am. Phys. Soc. 41, 1371 (1996)

${ }^{10}$ J. A. Snipes D. J. Campbell, T. C. Hender, M. von Hellerman, and H. Weisen, Nucl. Fusion 30, 205 (1990).
${ }^{11}$ R. J. La Haye, R. Fitzpatrick, T. C. Hender, A. W. Morris, J. T. Scoville, and T. N. Todd, Phys. Fluids B 4, 2098 (1992).

${ }^{12}$ T. C. Hender, R. Fitzpatrick, A. W. Morris, P. G. Carolan, R. D. Durst, T. Edington, J. Ferreira, S. J. Fielding, P. S. Haynes, J. Hugill, I. J. Jenkins, R. J. La Haye, B. J. Parnham, D. C. Robinson, T. N. Todd, M. Valovic, and G. Vayakis, Nucl. Fusion 32, 2091 (1992).

${ }^{13}$ R. J. La Haye, A. W. Hyatt, and J. T. Scoville, Nucl. Fusion 32, 2119 (1992).

${ }^{14}$ R. Fitzpatrick, Nucl. Fusion 33, 1049 (1993).

${ }^{15}$ Z. W. Ma, X. Wang, and A. Bhattacharjee, Phys. Plasmas 3, 2427 (1996).

${ }^{16}$ X. Wang and A. Bhattacharjee, Phys. Plasmas 4, 748 (1997).

${ }^{17}$ T. S. Hahm and R. M. Kulsrud, Phys. Fluids 28, 2412 (1985).

${ }^{18}$ X. Wang and A. Bhattacharjee, Phys. Fluids B 4, 1795 (1992).

${ }^{19}$ W. Park, D. A. Monticello, and R. B. White, Phys. Fluids 27, 137 (1984).

${ }^{20}$ F. L. Waelbroeck, Phys. Fluids B 1, 2372 (1989).

${ }^{21}$ J. W. Connor, S. C. Cowley, R. J. Hastie, T. C. Hender, A. Hood, and T. J. Martin, Phys. Fluids 31, 577 (1988).

${ }^{22}$ J. W. Connor, R. J. Hastie, and J. B. Taylor, Phys. Fluids B 3, 1532 (1991).

${ }^{23}$ H. P. Furth, J. Killeen, and M. N. Rosenbluth, Phys. Fluids 6, 459 (1963).

${ }^{24}$ E. N. Parker, J. Geophys. Res. 62, 509 (1957).

${ }^{25}$ P. A. Sweet, Electromagnetic Phenomena in Cosmical Physics (Cambridge University Press, New York, 1958), p. 123.

${ }^{26}$ P. H. Rutherford, Phys. Fluids 16, 1903 (1973).

${ }^{27}$ R. Fitzpatrick, Phys. Plasmas 1, 3308 (1994).

${ }^{28}$ F. Porcelli, Phys. Rev. Lett. 66, 245 (1991).

${ }^{29}$ L. Zakharov, B. Rogers, and S. Migliuolo, Phys. Fluids B 4, 3285 (1992). 